\title{
Vortex induced confinement and the IR properties of Green functions*
}

\author{
K. Langfeld, J.C.R. Bloch, J. Gattnar, H. Reinhardt \\ Institut für Theoretische Physik, \\ Universität Tübingen, D-72076 Tübingen, Germany \\ A. Cucchieri and T. Mendes ${ }^{\dagger}$ \\ IFSC São Paulo University \\ C.P. 369 CEP 13560-970, São Carlos (SP), Brazil
}

November 2, 2018

In order to observe the confinement of quarks and gluons in Yang-Mills theory, two conditions must necessarily be obeyed [1]: (i) there must be a long range order of certain un-physical degrees of freedom (violation of the cluster decomposition ); (ii) there must be a mass gap for physical excitations. Using Landau gauge where the gauge configurations are restricted to the first Gribov regime, it was firstly speculated by Gribov [2] that the divergence of the ghost propagator at zero momentum transfer signals confinement. Over the years, Zwanziger has put forward that the configurations which are relevant for confinement are close to the Gribov horizon[3]. A formal relation between confinement and the IR properties of confinement was advocated by Kugo and Ojima [4]: their approach is based on the assumption that a unique definition of the BRST charge operator exists. This assumption, however, is invalidated by the presence of Gribov copies.

In my talk, I will explore the relation between the IR properties of Green functions and confinement using $\mathrm{SU}(2)$ lattice gauge theory.

Recent investigations of the Dyson-Schwinger equations 朐, 6] have revealed that the ghost-propagator $G\left(p^{2}\right)$ and the gluon-propagator $D\left(p^{2}\right)$ satisfy remarkable scaling relations in the IR limit:

$$
G\left(p^{2}\right) \sim p^{-2-2 \kappa}, \quad D\left(p^{2}\right) \sim p^{-2+4 \kappa},
$$

\footnotetext{
*Talk presented by K. Langfeld at "Confinement V", Gargano, Italy, 10-14 Sept 2002.

${ }^{\dagger}$ Work supported by FAPESP, Brazil (Project No. 00/05047-5).
} 

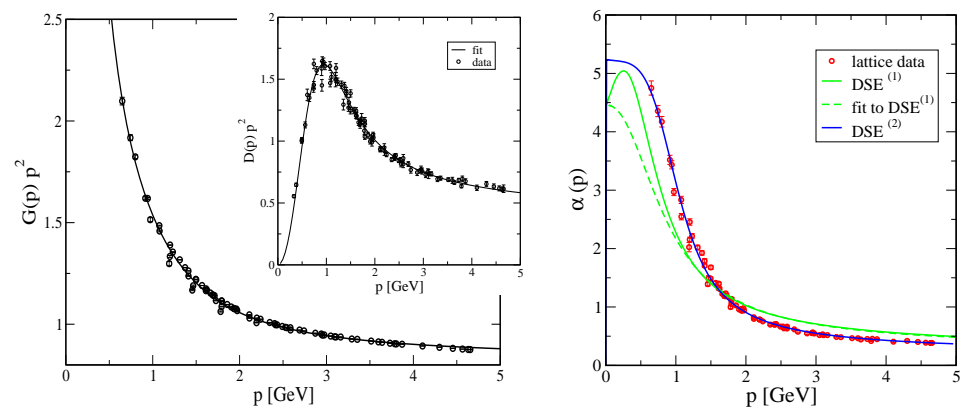

Figure 1: Ghost- and gluon-form factor (left), running coupling (right).

where $\kappa \in[0.5,1]$ depending on the truncations. Some of our lattice results for these propagators are shown in figure 1 (see also [7]). The lattice data are consistent with an IR diverging form factor with $\kappa \approx 0.5$. This finding is in agreement with a large scale volume study of the Adelaide group [8]. The IR scaling relation (11) implies that the running coupling $\alpha\left(p^{2}\right)=\alpha\left[p^{2} D\left(p^{2}\right)\right]\left[p^{2} G\left(p^{2}\right)\right]^{2}$ approaches a constant for $p \rightarrow 0$. In the present lattice simulations, we have not yet observed the flattening of the coupling at low momenta. Additional theory input is required to detect the fixed point value $\alpha(0)$. Two truncations of the DSE system are presently available: $\operatorname{DSE}^{(1)}$ [9], and a truncation scheme using a 2-loop inspired kernel and using $\kappa=0.5\left(\mathrm{DSE}^{(2)}\right)$ [10]. Using the latter DSE solution as an additional theory input, our lattice data are consistent with the fixed point value: $\alpha(0)=5.2 \pm 0.3$.

It was recently observed 11] that removing the percolating vortex cluster from the lattice configurations by hand implies quark de-confinement. At the same time, the IR divergence of the ghost form factor is removed (see figure 2). Here, we present for the first time the ghost form-factor $G(0, \vec{p})$ at temperatures well above the de-confinement phase transition (see figure 2). Using dimensional reduction, we know that the 3-dimensional reduced theory describing Yang-Mills theory at asymptotic temperatures, is in the confining phase. Hence, we expect that the ghost form-factor is still diverging for $\vec{p}^{2} \rightarrow 0$. Our lattice result meets with this expectation.

We argue that the removal of the percolating cluster of confining vortices restores cluster decomposition: the lattice study [11] shows that, in this case, the theory is converted in a non-confining theory whose ghost form-factor is regular in the IR limit. It was observed in the recent past 12] that the de-confinement phase transition at finite temperatures can be viewed as vortex de-percolation transition: considering a hypercube spanned by the time and 2-spatial axis, the vortex world lines stop percolating at $T_{c}$. Cluster de-composition is partially restored. At the same time, the spatial hypercube is still filled by percolating vortex world lines. As a result, the violation of cluster decomposition of the 3 -dimensional reduced theory by means of percolating vortex world lines is still 

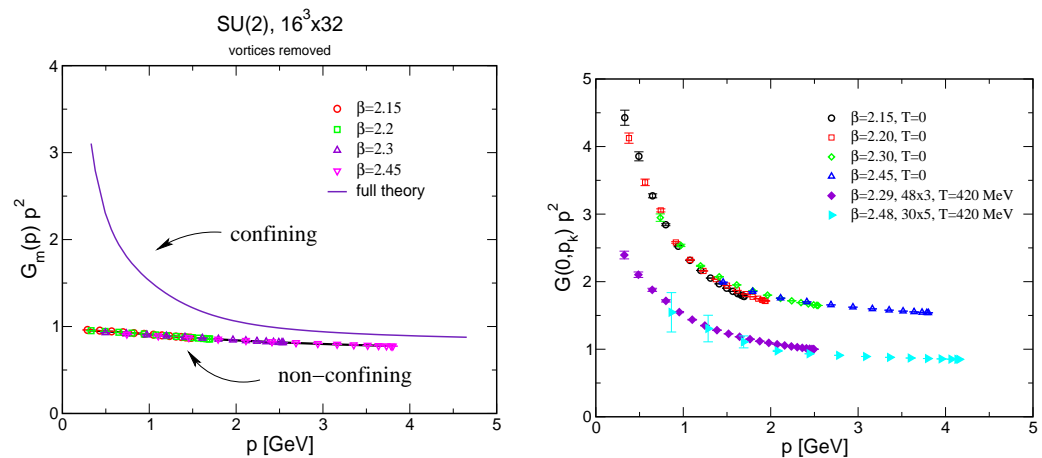

Figure 2: Ghost-form factor of the non-confining theory (left), Ghost-form factor at finite temperatures (right).

active (as requested for an explanation of the spatial string tension).

\section{References}

[1] A. Jaffe, E. Witten, "Quantum Yang-Mills Theory", The Millennium Prize Problems, http://www.claymath.org/prizeproblems/yangmills.htm.

[2] V. N. Gribov, Nucl. Phys. B 139, 1 (1978).

[3] D. Zwanziger, Nucl. Phys. B 378, 525 (1992).

[4] T. Kugo and I. Ojima, Prog. Theor. Phys. Suppl. 66, 1 (1979).

[5] L. von Smekal, R. Alkofer and A. Hauck, Phys. Rev. Lett. 79, 3591 (1997) L. von Smekal, A. Hauck and R. Alkofer, Annals Phys. 267, 1 (1998)

[6] D. Atkinson and J. C. R. Bloch, Phys. Rev. D 58, 094036 (1998)

[7] J. C. Bloch, A. Cucchieri, K. Langfeld and T. Mendes, hep-lat/0209040.

[8] F. D. Bonnet, P. O. Bowman, D. B. Leinweber, A. G. Williams and J. M. Zanotti, Phys. Rev. D 64, 034501 (2001)

[9] C. S. Fischer and R. Alkofer, Phys. Lett. B 536, 177 (2002)

[10] J.C.R. Bloch, in preparation.

[11] K. Langfeld, arXiv:hep-lat/0204025.

[12] K. Langfeld, O. Tennert, M. Engelhardt and H. Reinhardt, Phys. Lett. B 452, 301 (1999)

M. Engelhardt, K. Langfeld, H. Reinhardt and O. Tennert, Phys. Rev. D 61, $054504(2000)$ 